\section{Anti-cell antibody in macaques}

SIR - Infection of macaques by simian immunodeficiency viruses (SIV) leads to a disease similar to that produced by HIV in man. This simian system is widely used in the United States and Europe as a model for the development of vaccines against AIDS. There are several reports ${ }^{1-4}$ that inactivated vaccines completely protect against intravenous challenge with homologous SIV. It is widely assumed that, as with other viral vaccines, protection is mediated by immune responses to viral antigens in these vaccines. But, although the vaccines do induce high titres of antibodies to SIV, our attempts to correlate protection with titres of virus-neutralizing antibody or, indeed, any anti-SIV antibodies (T. Corcoran et al. and P. S. et al., in preparation) have been uniformly unsuccessful. Similar observations have been reported by others ${ }^{1,3,5}$ and were discussed in News and Views ${ }^{6}$.

We have demonstrated that inactivated purified virions or fixed SIVinfected cells are protective. During experiments to define the protective components in these vaccines and the minimum dose schedule, four macaques (Macaca fascicularis) were vaccinated with inactivated SIV-infected C8166 cells (group A) and four with uninfected cells (group B) at 0 and 4 weeks using Quil A as adjuvant. These animals were challenged at 6 weeks with 10 MID $_{50}$ of SIV intravenously. Three macaques in group A and two in group B were protected.

In view of this surprising result, the five protected animals were revaccinated with their respective vaccines at week 27 and rechallenged at week 29. Two of three in group $\mathrm{A}$ and one of two in group $\mathbf{B}$ were protected. In these experiments and on the nine other occasions when this challenge virus was used, four of four unvaccinated control macaques became infected. Thus, there was no significant difference in the protection induced by the two vaccines.

The uninfected cell vaccine did not induce antibodies to SIV that could be detected by ELISA or immunoblotting. As expected, all vaccinated animals made antibody against $\mathrm{C} 8166$ cells which was measured by ELISA on the day of challenge. But we did not expect to discover that the mean titre of the eight animals protected against challenge was tenfold higher than the mean of five animals which became infected, and that this difference was highly significant. The mean titre \pm standard error of protected group is $\log _{10} 3.49 \pm 0.07$ and of the infected group was $\log _{10} 2.42 \pm$ 0.08 , giving $P<0.0001$ by $t$-test.

In the light of this finding we reexamined sera from animals vaccinated with gradient- or column-purified virus as well as those given SIV-infected cells. Nine became infected following challenge and forty were protected. The mean titre of antibody to C8166 cells on the day of challenge in the protected group was $\log _{10} 3.39 \pm 0.09$ and in the unprotected group was $\log _{10} 2.39 \pm 0.10$, $P<0.0001$. Certain of the animals vaccinated with purified virus had higher titres to $\mathrm{C} 8166$ cells than those vaccinated with fixed SIV-infected cells. Similar results were obtained when CEM-4 cells were used as antigen and surface fluorescence was measured by flow cytometry; mean titre of protected animals was $\log _{10} 3.67$ \pm 0.12 , and of the unprotected groups was $1.75 \pm 0.09, P<0.0001$.

We are performing definitive experiments to confirm these observations, to determine the full extent of protection mediated by antibody to cellular components of the vaccine or challenge virus, and to define more specifically the target for this antibody. Our findings do not, of course, exclude the possibility of a virusspecific component in protection, and studies with recombinant antigens will eventually clarify this mechanism of immunity. In the meantime, we wish to publish our unexpected findings quickly macaques or HIV-1 in chimpanzees can evaluate their results in the light of this information.

Whatever the outcome, experiments in animal models have demonstrated that vaccines induce complete protection in vivo against challenge viruses which induce AIDS-like disease; our results highlight the importance of the SIVmacaque model in which protective mechanisms can be examined. We are not aware that antibody against cell components has been shown to protect in vivo against any other type of virus infection. Our results, if confirmed, may reveal another unique property of immunodeficiency viruses which requires explanation and may profoundly affect our understanding of such viruses and the way we strive to control them.

National Institute for Biological

E. J. Sтот̈*

Standards and Control,

Blanche Lane, South Mimms,

Potters Bar, Herts EN6 3QG, UK

*Other signatories: P. A. Kitchin, M. Page, B. Flanagan, L. F Taffs, W. L. Chan K. H. G. Mills, P. Silvera and A. Rodgers. Research funded by MRC.

1. Desrosiers, R. C. et al. Proc. natn. Acad. Sci. U.S.A. 86 6353-6357 (1989).

2. Murphy-Corb, M. et al. Science 246, 1293 (1989)

3. Carlson, J. R. et al. AIDS Res. hum. Retrovir. 6, 1239 1246 (1990)

4. Stott, E. J. et al. Lancet II, 1538-1541 (1990).

5. Murphy-Corb, M. el al. AIDS 5, 655-662 (1991).

6. Moore, J. \& Weiss, R. Nature 352, 376-377 (1991). so that others working with SIV in
Red Rectangle emission

SIR - P. J. Sarre in Scientific Correspondence ${ }^{1}$ has drawn attention to the near correspondence in wavelength between a group of diffuse interstellar absorption bands and some of the the discrete emission features observed in the Red Rectangle ${ }^{2}$, a coincidence that I have independently noted ${ }^{3}$. Sarre points out that the profiles of the emission features at wavelengths $\lambda \lambda 5,799,5,855$ and $6,615 \AA$ are typical of band heads formed by the contours of unresolved $R$-branch molecular rotational lines. I suggest that the more symmetric emission features at $\lambda \lambda 5,828$ and $5,880 \AA$, and a shoulder on the red wing of the $\lambda 6,615$ feature $^{2,4}$, correspond in each case to the blended contours of the $Q$ and $P$-branch rotational lines.

For a given kinetic tempertature, $T$, and a Boltzmann population of rotational energy levels ${ }^{5}$, the energy separation of the strongest $R$ - and $P$-branch rotational lines is given by $\left(8 k T B^{\prime} / h c\right)^{1 / 2}$, where $B^{\prime}$ is the rotational constant of the molecule in the excited electronic state. The measured separation of a given pair of contour peaks therefore provides an estimate of $B^{\prime}$, if the $P$ branch contour dominates the redward emission feature.

For a nebular temperature in the range $3,000-7,000$ kelvin, $B^{\prime} \sim 0.45$ $0.20 \mathrm{~cm}^{-1}$ for the $\lambda 5,799 / \lambda 5,828 \lambda 5,849 /$ $\lambda 5,880$ pairs of contours; for the $\lambda 6,615$ feature and its shoulder, $B^{\prime} \sim 0.10-0.14$ $\mathrm{cm}^{-1}$ over this range of temperatures. Such rotational constants are typical of carbon-chain molecules of about three to seven atoms in length, or small polycyclic aromatic hyrdrocarbons containing between one and three rings. These values certainly exclude $\mathrm{C}_{60}$ (as discussed by Sarre) and large polycyclic aromatic hydrocarbons as candidate molecules for the emission features.

The suggesion that carbon-chain molecules exist in the Red Rectangle is not new ${ }^{2}$. I have argued elsewhere ${ }^{3}$ that the abundance of carbon-chain molecules and small graphite flakes in the diffuse features.

Department of Physics and

S. J. FOSSEY

Astronomy,

University College London,

Gower Street.

London WC1E 6BT, UK

1. Sarre, P. J. Nature 351, 356 (1991)

2. Warren-Smith, R. F. Scarrott, S. M. \& Murdin, P. Nature 292, 317-319 (1981)

3. Fossey, S. J. thesis, Univ. London (1990)

4. Schmidt, G. D., Cohen, M. \& Margon, B. Astrophys. J. Lett. 239, L133-L138 (1980).

5. Danks, A. C. \& Lambert D. L. Mon. Not. R. astr. Soc. 174, 571-586 (1976) 